\title{
PERÍMETRO CEFÁLICO: POR QUE MEDIR SEMPRE
}

\author{
HEAD CIRCUMFERENCE: WHY ALWAYS MEASURE IT
}

Luzita M. L. Macchiaverni' \& Antonio A. Barros Filho²

\begin{abstract}
${ }^{1}$ Assistente; ${ }^{2}$ Docente - Departamento de Pediatria da Faculdade de Ciências Médicas - UNICAMP.
CorRespondêncIA: Luzita Maria Laranjeira Macchiaverni - Departamento de Pediatria da Faculdade de Ciências Médicas - UNICAMP; Rua Alexander Fleming, 181 - Cidade Universitária "Zeferino Vaz" - Caixa Postal: 6111 CEP: 13083-970 - Campinas - SP.
\end{abstract}

MACCHIAVERNI LML \& BARROS FILHO AA. Perímetro cefálico: Por que medir sempre. Medicina, Ribeirão Preto, 31: 595-609, out./dez. 1998.

RESUMO: Este trabalho procura revisar algumas observações relatadas na literatura, referentes ao perímetro cefálico, como as técnicas antropométricas, as curvas de referência, a tendência secular, sua relação com o crescimento cerebral, sua relação com a desnutrição precoce, com o desenvolvimento neuromotor e com algumas patologias da idade adulta, resgatando a pertinência de usá-lo, não só para avaliação nos três (3) primeiros anos de vida, mas, também, em idades posteriores, como indicador de agravos nutricionais no início da vida.

Os autores descrevem as características do crescimento cerebral, que diferentemente de outras partes do corpo, tem $83,6 \%$ de seu crescimento completado dentro do primeiro ( $\left.1^{\circ}\right)$ ano de vida. Assim sendo, o perímetro cefálico (P.C.) é uma medida antropométrica que pode fornecer dados sobre a nutrição ocorrida nos dois (2) primeiros anos de vida. Assinalando os períodos de maior vulnerabilidade do cérebro humano, e os insultos nutricionais, apontam as evidências das seqüelas da desnutrição precoce para o cérebro, como a redução da massa cerebral, redução do número de células gliais, de células granulosas cerebelares, de Purkinje e do tamanho das redes, dos padrões de ramificação e do número de sinapses. Apresentam estudos realizados em populações que sofreram desnutrição nos dois (2) primeiros anos de vida e que mostram prejuízo no potencial para o desenvolvimento neuromotor, que é agravado ou minimizado pelas condições ambientais.

Estudos recentes sugerem que a desnutrição precoce está correlacionada com o aumento das doenças cardiovasculares na idade adulta, conseqüente aos mecanismos de adaptação da gestante e do lactente à situação nutricional. O perímetro cefálico menor também é fator de risco para a instalação mais precoce e gravidade da doença de Alzheimer, em adultos predispostos.

Os autores recomendam que a medida do perímetro cefálico conste em estudos de avaliação de saúde e nutrição populacionais, ao lado de outras medidas antropométricas comumente usadas.

UNITERMOS: Cefalometria. Transtornos da Nutrição Infantil. Desenvolvimento Infantil. Saúde Pública. Antropometria.

\section{INTRODUÇÃO}

A antropometria é um método simples, universal, não invasivo e de baixo custo, usado para avaliação do tamanho, proporções e composição do corpo humano. Como o crescimento e as dimensões corporais, em todas as idades, refletem a saúde e o bem estar de indivíduos e populações, podem, então, as medidas antropométricas ser usadas como indicadores de saúde, performance e sobrevivência. Estudos recentes têm ampliado as aplicações da antropometria, para identificar as desigualdades econômicas e sociais, apontar qual população deverá receber intervenção e avaliar as respostas às intervenções sofridas ${ }^{(1)}$. 
Os indicadores mais utilizados e recomendados para a avaliação do estado nutricional, na infância são: peso por idade, estatura ou comprimento por idade, peso por altura, circunferência braquial, pregas cutâneas, triciptal e subescapular, e o índice de massa corporal. Outra medida eventualmente recomendada é o perímetro cefálico. Como o crescimento cerebral se dá predominantemente nos três (3) primeiros anos de vida, tal fato fez com que o estudo do perímetro cefálico tenha sido restrito a esse período e, de certa forma, negligenciado, existindo poucas investigações ou avaliações que o utilizem após os três anos de idade.

Alguns autores, entretanto, têm assinalado que sua medida, após os três anos de idade, seria de grande utilidade para detectar as condições de nutrição ocorridas precocemente $^{(2)} \mathrm{e}$, mais do que a estatura, $\mathrm{o}$ perímetro cefálico seria a medida mais adequada para detectar a desnutrição ocorrida nos primeiros anos de vida $^{(3)}$.

Sendo essa medida altamente correlacionada com o tamanho cerebral, ${ }^{(4 / 7)}$, vários estudos procuram esclarecer a influência da desnutrição grave e precoce no crescimento cerebral. Embora o cérebro apresente certa plasticidade frente aos insultos, e o desenvolvimento neuromotor dependa de fatores não somente fisiológicos, mas, também, do meio ambiente, é necessário ter melhor compreensão dos efeitos deletérios da desnutrição sobre o sistema nervoso, pois estes podem levar às mais graves seqüelas dos "sobreviventes" de uma má nutrição na infância.

Este trabalho procura revisar algumas observações relatadas na literatura, referentes ao perímetro cefálico, como as técnicas antropométricas, as curvas de referência, a tendência secular, sua relação com o crescimento cerebral, sua relação com a desnutrição precoce, com o desenvolvimento neuromotor e com algumas patologias da idade adulta, resgatando a pertinência de usá-lo, não só para avaliação nos três (3) primeiros anos de vida, mas, também, em idades posteriores, como indicador de agravos nutricionais no início da vida.

\section{PERÍMETRO CEFÁliCo}

O perímetro cefálico é descrito como a circunferência "frontoccipital" ou como a circunferência "Frankfurt Plane", correspondendo ao perímetro cefálico máximo.

Para a sua medida, SMITH, $1977^{(8)}$, recomenda que a fita seja posicionada sobre a proeminência occipital e sobre o arco das sobrancelhas, lembrando que $1 \mathrm{~mm}$ de espessura do couro cabeludo aumenta o perímetro cefálico em $6 \mathrm{~mm}$. CAMERON, 1984(9), preconiza, para crianças maiores de dois (2) anos, que o sujeito seja medido em pé, com o lado esquerdo em frente ao observador. Os braços devem estar relaxados. É mais fácil, se o observador se posicionar no mesmo nível da criança. A fita, de material inextensível, é então passada ao redor da cabeça, da esquerda para direita, e cruzada na frente do observador. Usando o dedo médio, pressiona-se a fita sobre a testa, movendo-a para cima e para baixo, determinando a parte mais anterior da cabeça. Isso feito, repete-se a manobra para determinar a porção mais posterior da região occipital. Uma vez determinados os dois pontos, a fita é puxada para comprimir o cabelo, e a leitura é feita, considerando-se a última unidade de medida completa. JELLIFFE \& JELLIFFE, $1989^{(6)}$, recomendam fixar a cabeça da criança, colocar a fita firmemente ao redor do osso frontal sobre o sulco supra-orbital, passando-a ao redor da cabeça, no mesmo nível de cada lado, e colocando-a sobre a proeminência occipital, máxima.

Embora os autores difiram nas descrições quanto ao procedimento, elas têm em comum medir o perímetro cefálico, máximo, o que possibilita a comparação entre diferentes estudos.

Nos países em desenvolvimento, quando as taxas de mortalidade infantil e proporcional caem a níveis baixos, estes deixam de ser indicadores sensíveis da qualidade de saúde de uma comunidade. Ao mesmo tempo, os estudos antropométricos, realizados periodicamente, crescem em importância para essa avaliação ${ }^{(10,11)}$.

Representando basicamente o crescimento do sistema nervoso central, o PC vem sendo rotineiramente usado para seguimento individual de crianças que apresentem aumento muito grande da cabeça, como nos casos de hidrocefalia, ou diminuição muito acentuada nas microcefalias, e associado às neuropatologias.

Seu uso, como indicador nutricional, tem se restringido a algumas situações nas quais não são conhecidas as idades, onde é relacionado com outros indicadores, como propuseram KANAWATI \& MACLAREN, $1970^{(12)}$, (o quociente perímetro braquial - perímetro cefálico), afirmando que entre três (3) e quarenta e oito (48) meses, os valores inferiores a 0,31 seriam representativos de desnutrição.

As medidas esqueléticas nas crianças, como altura ou comprimento, medida do segmento inferior, 
altura sentada, refletem o "status" nutricional passado. Mas a interpretação desses dados é influenciada por vários fatores, como a idade, o momento e a intensidade do episódio de má nutrição e como a criança viveu após esse período. Por exemplo, uma criança com baixa estatura aos cinco (5) anos, pode ser o resultado de um único episódio de má nutrição, ou de múltiplos, durante qualquer período anterior. Há evidências de que o perímetro cefálico pode refletir deficiências nutricionais que ocorreram no início da vida, ${ }^{(13,14,15)}$ podendo ser um indicador muito útil no planejamento de ações que visem minimizar ou prevenir os efeitos da má nutrição precoce.

As curvas de referência para o perímetro cefálico são importantes para o seguimento de crianças com problemas neurológicos e para uma melhor e mais completa avaliação nutricional, dos lactentes, segundo recomendações da Organização Mundial da Saúde ${ }^{(16)}$ e, na idade escolar, para avaliar o passado nutricional $^{(15)}$.

NELHAUS, $1968^{(17)}$, elaborou curvas do crescimento do perímetro cefálico, segundo sexo e idade, do nascimento até os dezoito (18) anos, baseando-se em estudos publicados de 1948 a 1965. Nas curvas, prevalecem crianças de baixo estrato social, originadas de estudos transversais e longitudinais de diferentes grupos étnicos. Conclui que não existem diferenças significativas quanto à raça, nacionalidade ou situação geográfica, considerando "normal" a medida do perímetro cefálico que estiver entre os percentis dois (2) e noventa e oito (98), correspondendo, esse intervalo, a +/- 2DP.

MEREDITH, $1971^{(18)}$, revendo as curvas de crescimento de perímetro cefálico de vários estudos, encontrou uma variabilidade nas médias, dependendo da etnia e situação geográfica, obtendo dados conflitantes com os referidos por NELHAUS, $1968^{(17)}$. Demonstra variações de mais de $1 \mathrm{~cm}$ ao nascimento, de $2,5 \mathrm{~cm}$ entre crianças e de até $4 \mathrm{~cm}$, entre grupos de adultos. Dentro de cada grupo, os sujeitos de sexo masculino apresentam médias superiores às das pessoas de sexo feminino. A magnitude das diferenças entre os sexos aumenta durante o primeiro $\left(1^{\circ}\right)$ ano de vida, tende a diminuir durante a infância, e aumenta novamente a partir da adolescência e idade adulta. Essas diferenças persistem em estudos feitos em diferentes países e etnias.

BABSON, $1970^{(19)}$, a partir de um estudo longitudinal de crianças de baixo peso ao nascer, do nascimento até o primeiro $\left(1^{\circ}\right)$ ano de vida, observou que, em relação ao perímetro cefálico, os prematuros, após um atraso inicial, aumentam a velocidade de crescimento aproximando-se e, temporariamente, ultrapassando o esperado para o crescimento fetal; em seguida, a curva segue os padrões normais. Para as crianças com retardo de crescimento intra-uterino, a curva de perímetro cefálico mantém-se abaixo da média esperada para a idade. Estudos posteriores, demonstram as mesmas tendências ${ }^{(20)}$.

TANNER apud SMITH, $1977^{(8)}$, analisou o comportamento do perímetro cefálico em crianças inglesas, em estudo longitudinal, publicado em 1973, do nascimento até os dezesseis (16) anos. O autor construiu tabelas para o sexo masculino e feminino, nas quais estabeleceu a média e o desvio padrão do perímetro cefálico em relação à idade. Essas tabelas são amplamente usadas como referência em estudos internacionais.

ROCHE et al., $1986^{(21)}$, por meio de um estudo de seguimento e medidas seriadas do perimetro cefálico, obtidas entre 1928 e 1967, apontam para fatores que, além do tamanho do cérebro, contribuem para a determinação da medida do perímetro cefálico, como a espessura do couro cabeludo, dos ossos cranianos e, mais tardiamente, o aumento da protuberância occipital externa e da região frontal, áreas que coincidem com os locais de medida do P.C. Dessa forma, é necessário cuidado ao inferir, após os primeiros anos de vida, a relação estreita de P.C. e tamanho de massa cerebral. Descrevem o estirão que ocorre durante a puberdade e que é, em parte, decorrente do aumento do encéfalo, pois há uma aceleração do crescimento da base do crânio. Mostram que há uma baixa correlação entre o estirão da estatura com o estirão cerebral, ressaltando que a puberdade não é um evento que ocorre em um só momento, apresentando uma série de transformações que ocorrem em diferentes tempos, dependendo das mudanças hormonais e dos órgãos atingidos. ROCHE et al. $1987^{(5)}$, estabeleceram curvas do perímetro cefálico do nascimento até os dezoito (18) anos, com os dados de um estudo longitudinal de crianças que haviam participado do Fels Longitudinal Study, do qual derivaram as curvas de crescimento do perímetro cefálico, do nascimento até os trinta e seis (36) meses, elaboradas pelo National Center for Health Statistics (NCHS). Desse estudo, os autores utilizaram uma amostra de oitocentas e oitenta e oito (888) crianças brancas e supostamente sadias. Nessas curvas, o estirão cerebral da puberdade não aparece, porque é pequeno e ocorre em dife- 
rentes idades entre os indivíduos. Os autores apresentam dados discretamente maiores que a os da curva de Nelhaus, e sugerem ser o reflexo do "status" sócio-econômico, da tendência secular ou dos diferentes métodos de medida.

ISHIKAWA et al., 1987(22), elaboraram curvas de crianças japonesas nascidas em Nagoya, de 1964 a 1981, do nascimento aos quinze (15) anos, por meio de um estudo longitudinal, encontrando diferenças menores que a média para o P.C. dos meninos em relação à curva de NELHAUS e de um estudo realizado em Oxford ${ }^{(23)}$. O estudo mostra estirão cerebral durante a adolescência e que este ocorre mais precocemente nas meninas do que nos meninos (onze (11) e catorze (14) anos, respectivamente). Os autores afirmam que a diferença está associada à maturação sexual, e neste estudo, coincide com o estirão para estatura.

JAFFE et al., 1992(24), enfatizam que estudos feitos em crianças, do nascimento até dois anos de idade, mostram variabilidade na aceleração ou desaceleração do crescimento, em relação ao perímetro craniano, em vários momentos. A aceleração ocorre antes da desaceleração e é freqüente a mudança de canal de crescimento, fenômeno que ocorre também para peso e estatura. Portanto, crianças saudáveis, com adequado desenvolvimento neuropsicomotor e que, durante o primeiro ano de vida, apresentem oscilação de canal de crescimento do P.C., devem ser observadas clinicamente, antes de se instituir qualquer investigação laboratorial ou de imagens. Aos treze (13) meses, $95 \%$ das crianças já apresentam seu canal de crescimento estabilizado.

No Brasil, as curvas mais utilizadas são as de MARCONDES \& MARQUES, $1983^{(25)}$, que contemplam as idades desde o período do nascimento aos trinta e seis (36) meses, por sexo.

Em todas a curvas citadas, o perímetro cefálico do sexo masculino é maior do que o do feminino, do nascimento aos dezoito (18) anos de idade. Assim como o tamanho corporal, o desenvolvimento muscular e esquelético e a distribuição de gordura, o perímetro cefálico é uma característica sexual secundária, fazendo parte do dimorfismo sexual, que tem como base os diferentes genótipos: masculino (XY) e feminino (XX). A medida de um parâmetro corporal reflete a interação genética e ambiental e a desnutrição pode alterar as diferenças entre os caracteres sexuais secundários. Os meninos apresentam taxa metabólica superior e maior velocidade de crescimento e, portanto, são mais susceptíveis a situações adversas ${ }^{(26)}$.
Quanto à etnia, WINGERD; SHEN \& SOLOMON, $1971^{(27)}$, estudando altura, peso e perímetro cefálico, em uma amostra de quinze mil (15.000) crianças da Califórnia, durante os dois primeiros anos de vida, compararam as médias de crescimento entre crianças brancas e negras, não encontrando diferenças significativas, sugerindo ser dispensáveis curvas específicas para brancos e negros que vivam nas mesmas condições sócio-econômicas.

DUNCAN; SMITH \& BRIESE, 1979(28), em um estudo de quatro mil, cento e sessenta e sete (4.167) escolares de sobrenomes hispânicos, dois mil, trezentos e vinte e dois (2.322), não hispânicos, sugerem que o retardo do crescimento (peso, altura e perímetro cefálico) das crianças de origem hispânica é muito mais um reflexo da situação sócio-econômica que da origem étnica ou genética.

\section{TENDÊNCIA SECULAR}

A tendência secular do crescimento, tanto na população infantil como na adulta, possibilita analisar o efeito das mudanças das condições de vida e saúde no tempo. Tendência secular positiva ou em ascensão vem sendo constatada em diversos países, e tem sido imputada às melhorias nas condições ambientais ${ }^{(29)}$.

Tendência secular do crescimento pode ser considerada como uma mudança no padrão de desenvolvimento somático, em crianças em uma determinada população, de uma geração para outra ${ }^{(30)}$.

Como TANNER, 1987(31), afirmou, "o crescimento é um espelho das condições da sociedade", então a tendência secular é um importante instrumento para avaliar as mudanças nutricionais, higiênicas e de saúde de uma população.

Tendências seculares são bem documentadas para idade da menarca (avanço ou retardo), do estirão da puberdade, para estatura e peso. Mas têm-se observado mudanças em outros valores antropométricos, como: comprimento das pernas, altura sentada e outras dimensões esqueléticas. Também são notadas alterações nos níveis de hemoglobina, na bioquímica sangüínea, idade óssea e no desenvolvimento dos dentes. Quanto à cabeça, há uma tendência secular à "debraquicefalização", isto é, um leve ou nenhum aumento no comprimento e uma diminuição na largura $(1,5 \mathrm{~mm} /$ década), verificada em trabalhos realizados na Bélgica, entre 1960 e 1980, e confirmados em estudos posteriores, realizados na Alemanha e na República Checa ${ }^{(30)}$. 
ROCHE et al., 1986 ${ }^{(21)}$, em um estudo longitudinal, em cento e dois (102) meninos e cento e nove (109) meninas, acompanhadas do nascimento aos dezoito (18) anos, demonstram que há evidência de tendência secular na mudança dos padrões de crescimento, quando comparam dados de 1928 e de 1967. Interessante é que, apesar disso, não encontrou mudança significativa do tamanho inicial (ao nascimento) e aos dezoito (18) anos.

ISHIKAWA et al., 1987(22), encontraram tendência secular positiva nas crianças japonesas nascidas em Nagoya (1964-1981), através de um estudo longitudinal em uma amostra de novecentas e vinte e seis (926) crianças, seguidas do nascimento aos quinze (15) anos de idade. O perímetro cefálico de ambos os sexos foi maior quando comparado com curvas obtidas em estudos anteriores, realizados em 1902 e 1930.

OUNSTED et al., $1985^{(23)}$, apresentam dados de um estudo longitudinal de medidas do P.C., do nascimento aos sete (7) anos, em que enfatizam a tendência secular positiva, quando esses dados são comparados com estudos anteriores. Referem que, quanto ao peso e estatura das crianças do estudo, as médias foram semelhantes às encontradas em gerações anteriores, mas, o P.C. foi maior, indicando que há tendência secular positiva, que foi evidente durante a primeira metade do século, continuando na década de setenta (70). A curva mais baixa obtida foi de crianças londrinas, em 1926.

DAVIES; LEUNG \& LAU $1986^{(32)}$, estudaram as medidas de perímetro cefálico de dois mil e quinhentos (2500) escolares de Hong Kong, de sete (7) a dezoito (18) anos, e compararam com curvas de dados coletados entre 1961 e 1965, demonstrando clara tendência secular positiva. Os autores afastam a possibilidade de erros nas medidas (empregaram o mesmo método) e o estudo foi realizado na mesma região. Diante dessa tendência, os autores colocam algumas questões como:

"O cérebro está sendo melhor nutrido atualmente, do que em décadas anteriores?"

"Há alguma associação neuropsicológica ainda não conhecida?"

Devido às diferenças de padrões de crescimento do perímetro cefálico, encontradas em vários estudos, alguns autores, como OUNSTED, $1985^{(23)}$, e ISHIKAWA $1987^{(22)}$, recomendam que os gráficos devam ser atualizados em cada geração e regionalizados.

\section{CRESCIMENTO CEREBRAL E NUTRIÇÃO}

O cérebro apresenta velocidade de crescimento muito diferente do crescimento geral de um indivíduo. O perímetro cefálico, que reflete o tamanho do encéfalo, aumenta de forma rápida durante o primeiro ano de vida, quando chega a atingir cerca de $83,6 \%$ do tamanho do adulto ${ }^{(33)}$. Após o primeiro ano de vida, embora a medida não seja usual, o perímetro cefálico aumenta suavemente até os dezoito (18) anos ${ }^{(17,34)}$. Após o terceiro $\left(3^{\circ}\right)$ ano, apesar de lento, o crescimento reflete $25 \%$ do volume cerebral ${ }^{(21)}$. A desnutrição grave, nos primeiros anos, pode comprometê-lo, determinando valores, no PC, inferiores aos esperados para a idade ${ }^{(2,33,35 / 39)}$.

DOBBING, 1981 ${ }^{(40)}$, investigando desnutrição e dano cerebral, em estudos experimentais com animais e em estudos de cérebros humanos, demonstrou que uma característica importante da restrição alimentar, no crescimento cerebral, é de que não há destruição de tecidos, mas, sim, uma falha seletiva. Somente com uma contagem histológica é possível determinar alguma anormalidade. Há um extremo contraste entre a delicada suscetibilidade e vulnerabilidade de um cérebro em crescimento e um cérebro adulto. Em certos estágios do desenvolvimento, agravos nutricionais, moderados, que podem ocorrer mesmo em países desenvolvidos, são suficientes para produzir déficit permanente. As estruturas atingidas na época do insulto são as que estão em fase de crescimento acelerado. Muitas etapas do desenvolvimento cerebral têm somente uma oportunidade para ocorrer em um momento determinado. Caso as condições não sejam favoráveis para que esse evento ocorra naquele momento, a oportunidade estará perdida para sempre. Como o desenvolvimento do cérebro é extremamente intrincado, há poucas oportunidades de compensar esse déficit, quando comparado ao crescimento de outros tecidos. O período de crescimento acelerado é chamado estirão. Em todos os mamíferos, o estirão cerebral se inicia ao término da multiplicação neuroblástica, caracteriza-se pela multiplicação glial, crescimento dendrítico, formação de sinapses e termina com a fase rápida de mielinização. A restrição do crescimento durante o estirão, pode acarretar lesões, pois este é o período de maior vulnerabilidade cerebral. A desnutrição, levando a uma desaceleração do crescimento, está associada a um cérebro menor que a média, e, por vezes, a uma verdadeira microcefalia. Nem todas as partes se reduzem na mesma intensidade. Por exemplo, o cerebelo se reduz bem 
mais que outras partes. Isso porque ele cresce muito rápido. Tal fato está associado com a presença de descoordenação, observada nos ratos, em estudos experimentais. Observa-se, também, ausência de neurônios granulares e perda de neurônios da camada cortical no cerebelo. A deficiência de lipídios é maior do que a esperada para o tamanho do cérebro e o número de sinapses é substancialmente reduzido ${ }^{(40)}$.

Sabe-se que, após um episódio de desnutrição grave, o cérebro de ratos apresenta mudanças neuroanatômicas permanentes, que compreendem a diminuição do peso cerebral, principalmente do cerebelo, redução do número de células gliais e de células granulosas cerebelares, diminuição do quociente entre células granulosas e de Purkinje, deficiências e alterações nas espinhas dendríticas, do tamanho das redes e dos padrões de ramificação ${ }^{(41)}$. Além disso, os ratos apresentam diminuição na capacidade de resolver problemas ${ }^{(42)}$.

O cérebro humano é mais vulnerável aos insultos, em dois períodos: o primeiro, e menos comum, entre dez (10) a dezoito (18) semanas de gestação, no qual ocorre a multiplicação neuronal que pode ser afetada por fatores como exposição a radioatividade, bem documentada em crianças que nasceram de mães sobreviventes de Hiroshima e Nagasaki, ${ }^{(40)}$, infecções virais, como rubéola, anomalias cromossômicas, erros metabólicos como altos níveis de fenilalanina no plasma materno, medicamentos ingeridos pela mãe, como os esteróides, que, em uso prolongado, reduzem a divisão celular; o segundo, e mais comum, conhecido como crescimento posterior, ou estirão, se inicia na metade do período gestacional, prolongando-se até aproximadamente o décimo-oitavo $\left(18^{\circ}\right)$ mês pós-natal, sendo este, portanto, um dos maiores períodos de vulnerabilidade. É importante salientar que não mais que $1 / 6$ do estirão ocorre no período fetal, e portanto não menos que $6 / 7$ do estirão cerebral é pós-natal, e nos dois primeiros anos de vida ${ }^{(40)}$.

Devido aos avanços tecnológicos e um melhor conhecimento do RN de baixo peso ao nascer, a sobrevida aumentou e muitas investigações, procuram elucidar o padrão de crescimento dessas crianças. BJERRE, $1975^{(43)}$, em um estudo longitudinal, não constatou diferenças significativas em relação ao PC em crianças com baixo peso, saudáveis, comparadas com as do controle (>2.500g), após cinco (5) anos de seguimento. OUNSTED et al., 1982(44), encontraram diferenças significativas em peso, estatura e perímetro cefálico entre crianças com baixo peso ao nascer, comparadas com RN grandes para a idade gestacio- nal, após quatro (4) anos de seguimento, mostrando padrões de crescimento bastante diversos, correlacionados ao tamanho ao nascer. SANN et al., $1986^{(45)}$, comparando grupos de prematuros com peso adequado e desnutridos intra-útero, encontrou um percentual significativamente alto de crianças com microcefalia, aos cinco (5) anos de idade, entre os que sofreram desnutrição intra-uterina, principalmente nas crianças a termo, com peso inadequado, sugerindo que o insulto nutricional intra-útero leva a um menor P.C. RIZZARDINI et al., 1991 ${ }^{(46)}$, estudando recém-nascidos de muito baixo peso, adequados para a idade gestacional e saudáveis, através de um estudo de seguimento até os três (3) anos de idade, não encontraram diferenças, ao final do estudo, quanto ao P.C., quando comparados aos que nasceram com peso normal. HACK et al.,1993(47), investigando o crescimento de crianças com muito baixo peso ao nascer, em um estudo de seguimento de oito (8) anos, encontraram um P.C. significativamente menor, quando comparados com crianças que nasceram com peso normal. Os autores enfatizam a alta morbidade que as crianças de muito baixo peso apresentam no pós-natal como um dos fatores de falha no crescimento.

XAVIER et al., $1995^{(48)}$, em estudo longitudinal de recém-nascidos pré-termo, adequados para a idade gestacional (RNTAIG), mostram que, com assistência e aporte nutricional adequados, as curvas de crescimento (peso, comprimento e perímetro cefálico) atingem, em torno da quadragésima primeira $\left(41^{\mathrm{a}}\right)$ semana, valores semelhantes aos encontrados por LUBCHENCO, 1963 ${ }^{(49)}$, concluindo que os RNTAIG apresentam bom prognóstico, quando em ambiente adequado, em tempo relativamente rápido.

Portanto, o baixo peso ao nascer é um fator de risco, porém não determinante para um P.C. inadequado em idades posteriores. As causas da desnutrição intra-uterina, como as infecções e as anomalias congênitas, a hipóxia e distúrbios metabólicos ${ }^{(50)}$ são fatores que, associados, podem determinar falhas no crescimento.

Para BHARGAVA et al., 1995(20), que estudaram recém-nascidos de baixo peso até catorze (14) anos de idade, as crianças prematuras com peso adequado, apresentaram melhor crescimento que as desnutridas intra-útero. Os autores, baseados em estudos experimentais, sugerem que o insulto nutricional, ocorrendo em períodos críticos, prejudicam o desenvolvimento hipotalâmico, programando um permanente baixo nível de atividade, o que explicaria o déficit no crescimento aos catorze (14) anos. 
STOCH \& SMYTHE, $1976^{(3)}$, foram os primeiros, em 1955, a formular a hipótese de que o cérebro humano poderia ser um órgão vulnerável aos efeitos da desnutrição, durante os dois primeiros anos de vida.

Os trabalhos, resumidos na Tabela $I^{(3,7,14,36,51 / 57)}$, são unânimes em apontar a desnutrição precoce como causa de um P.C. menor que a média esperada para a idade. Como as populações desnutridas, em sua maioria, pertencem aos estratos sócio-econômicos menos favorecidos, a desnutrição encontra-se, também, associada à maior morbidade.

A diminuição do perímetro cefálico está diretamente correlacionada com a desnutrição provocada por moléstias crônicas que incidem nos primeiros anos de vida. Nos países em desenvolvimento, a doença diarréica, freqüente no primeiro ano de vida, leva a um P.C. abaixo da média ${ }^{(58)}$. Problemas pulmonares como fibrose cística e a broncodisplasia, freqüentes nos prematuros, também podem levar a um P.C. menor $^{(59,60)}$. A doença renal crônica, em lactentes, leva à microcefalia e a um grave comprometimento neurológico, sendo a desaceleração do crescimento do P.C. usada como parâmetro para indicação de diálise ${ }^{(61)}$. APPLETON, $1990^{(62)}$, mostra a importante redução do PC, sofrida por crianças que receberam tratamento por radioterapia, acompanhada de declínio no desenvolvimento cognitivo.

Os autores salientam que a medida do P.C. é o parâmetro mais sensível aos insultos nutricionais, que ocorrem antes dos dois anos de idade ${ }^{(14,58)}$.

\section{DESNUTRIÇÃO, DESENVOLVIMENTO CERE- BRAL E PERFORMANCE INTELECTUAL}

Ao considerarmos o perímetro cefálico como um parâmetro que reflete o crescimento do próprio encéfalo, e sendo ele afetado nos processos de desnutrição precoce, grave e de longa duração $0^{(14,36,56)}$, as crianças que passaram por injúrias nutricionais desse tipo carregam as sequielas para a idade adulta. Tal fato se reveste de importância, quando investigações demonstram que a desnutrição precoce e grave pode interferir na performance intelectual dos indivíduos afetados ${ }^{(63,64)}$ e que o P.C. tem se mostrado como um preditor do futuro intelectual $^{(65,66)}$.

ROSADO et al., 1989(67), em ampla revisão de estudos sobre o crescimento craniano de recém-nascidos e lactentes, procuram analisar e correlacionar o P.C. com dados antropométricos e variáveis biológicas e sociais maternas. Os autores concluem que o conhecimento de fatores que podem influenciar o de- senvolvimento cerebral da criança, é importante não só para estabelecer comparações em diferentes populações como para detectar aqueles que podem comprometer o neurodesenvolvimento e a potencialidade intelectual.

Considerando estudos experimentais sobre carências nutricionais e seus efeitos deletérios sobre o sistema nervoso, foram realizadas numerosas pesquisas, tentando quantificar a ação da desnutrição, correlacionada ou não com diminuição do PC, no desenvolvimento neuropsicomotor de crianças afetadas. Alguns dos trabalhos estão resumidos na Tabela II ${ }^{(63,66,68 / 71)}$.

É extremamente difícil definir o papel da desnutrição no desenvolvimento mental. A natureza heterogênea das deficiências nutricionais, poderia explicar uma das dificuldades. Além dos déficits protéicos e energéticos, pode haver deficiências de muitos outros nutrientes, como oligoelementos e vitaminas.

A deficiência de iodo é um exemplo clássico de lesão do SNC, levando a atraso mental ${ }^{(42)}$. Existem estudos, sugerindo que a deficiência de ferro também afeta o desenvolvimento cerebral, traduzindo-se esse efeito sobre mudanças de comportamento que são revertidas após suplementação, em crianças com anemia ferropriva ${ }^{(72)}$. Experimentos, em animais, têm demonstrado que a hipovitaminose A, assim como a hipervitaminose pode bloquear a circulação do líquido cérebro-espinal, causando hipertensão intracraniana ${ }^{(73)}$.

Estudos experimentais, em animais, nos quais as variáveis podem ser rigorosamente controladas, a correlação entre desnutrição, desaceleração do crescimento cerebral e seqüelas neurológicas é bem estabelecida. A extrapolação desses dados para o ser humano não pode ser feita sem considerar que as deficiências nutricionais variam em gravidade e duração, desde episódios graves e relativamentes breves, a restrições menos graves que se prolongam desde o crescimento intra-uterino e ao longo de toda infância. As crianças que podem ser afetadas em diferentes fases de seu desenvolvimento, também são expostas a diferentes ambientes sócio-culturais e econômicos e é muito provável que todos esses fatores modifiquem os efeitos da desnutrição ${ }^{(42)}$.

É evidente que ante o insulto nutricional, o organismo se comporta com muito mais plasticidade do que sugere o conceito de período crítico, como mostram os estudos de intervenção ${ }^{(71,74)}$. Quando as condições ambientais satisfazem as necessidades fisiológicas e emocionais, o organismo pode alcançar os limites do desenvolvimento normal. Por outro lado, a desnutrição é um fator de risco para o desenvolvimento; 
Tabela IA - Sumário de estudos que correlacionam desnutrição e perímetro cefálico

\section{Autores}

Métodos
Resultados
Conclusões
Winick \& Rosso ${ }^{(36)}$

(1969) Chile, NY

Malina et al. ${ }^{(14)}(1975)$

Guatemala

Stoch \& Smythe ${ }^{(3)}$

(1976) Cidade do

Cabo África do Sul

Lechtig \& Klein ${ }^{(51)}$

(1979) Guatemala

Desai et al. ${ }^{(52)}(1981)$

Brasil
Estudo do cérebro de crianças
que morreram no $1^{\circ}$ ano de vida: marasmáticas ( $\mathrm{N}=9)$ - "normais" $(\mathrm{N}=10)$.

Determinação: do P.C., peso do cérebro, quantidade de DNA e de proteína.

Estudo longitudinal misto. $\mathrm{N}=1119$ do nascimento aos 7 anos.

Crianças da Guatemala, onde a desnutrição é endêmica.

Efetuadas 5012 medidas do P.C., e comparadas com estudo realizado em Denver (população nutrida).

Estudo longitudinal. Amostra $\mathrm{N}=20$ O P.C. foi significantemente me(9 masc. e 11 fem.), idade mé- nor entre os casos índices, quandia=16 anos. Os adolescentes da do comparado com o do grupo amostra sofreram desnutrição durante 0 1ำ ano de vida (marasmo). Grupo controle $\mathrm{N}=40$, do mesmo nível sócio-econômico, sem desnutrição precoce. Antropometria: P.C., altura e peso. Testes: Bender, Figura humana.

Estudo de intervenção S.N. (suplementação nutricional). 2 vilas recebem S.N. com alto nível calórico e energético.

2 vilas recebem S.N. de baixo nível.

Casuística: gestantes e lactentes 0-12m.

Antropometria: PC, altura, peso, circunferência braquial.

Estudo transversal comparativo. Amostra: $\mathrm{N}=455$ crianças faveladas. Idade: 1-16 anos Controle: $\mathrm{N}=475$ crianças de NSE ** elevado.

Avaliação: peso, altura, P.C., circunferência braquial prega triciptal e teste cicloergométrico. as crianças sem S.N. rença significativa. po de controle. A performance física foi menor. nômica.
As crianças marasmáticas apre- Durante o primeiro ano de vida, o sentaram: P.C.-2DP, peso do P.C. é muito útil, porque reflete cérebro, reduzido proporcional- acuradamente os padrões de dimente ao P.C., DNA reduzido visão celular do cérebro de criantanto quanto o P.C. e, em 3 ca- ças normais e desnutridas. sos, mais afetado.

As crianças da Guatemala apresentaram P.C. significantemente ser usado para estimar o retardo diferenças eram pequenas ao primeiros anos.

nascimento, mas já estavam bem estabelecidas aos 2 anos. Aos 5 anos o P.C. indicava a época do início da desnutrição. controle. Quanto à altura, a dife- dem somente ser explicados por rença diminuiu com a idade. Nos uma disfunção cerebral, orgânitestes, o grupo controle mostrou ca, central. melhor performance.

Os autores sugerem que as alterações são seqüelas da desnutrição grave e precoce.

As crianças das vilas com S.N. A altura, o peso e o P.C. são apresentaram: P.C. $>1,1 \mathrm{~cm}$; altu- bons indicadores para avaliação ra $>1,0 \mathrm{~cm}$ e peso $>220-430 \mathrm{~g}$ que nutricional de 0-12 meses.

A suplementação melhora o cresQuanto à circunferência braquial, cimento quando: fornecida às nuaos 12 meses, não houve dife- trizes, no $1^{\circ}$ semestre, e fornecida ao lactente no $2^{\circ}$ semestre.

Todas as medidas antropométri- Os autores comentam os resultacas das crianças faveladas foram dos, relacionando-os à desnutriinferiores às das crianças do gru- ção precoce, que continua na adolescência, e à situação sócio-eco- 


\section{Tabela IB - Sumário de estudos que correlacionam desnutrição e perímetro cefálico}

\section{Autores Métodos}

Grantham-McGregor et Estudo longitudinal. al. $^{(53)}$ (1982) Jamaica

Dagan et al. ${ }^{(54)}$ (1983)

Deserto de Negev Israel

Serenius et al. ${ }^{(55)}$

(1988) Arábia Saudita

Ivanovic et al. ${ }^{(56)}(1990) \quad$ Estudo transversal descritivo.

Chile

Wright et al. ${ }^{(57)}(1992)$

Oxford

Ivanovic et al. ${ }^{(7)}(1995)$

Chile das. entre 1975-1976. Development Scales). tatura.

Estudo transversal comparativo. judias. Idade: 0-12 m. rentes.

Europa. mentos.

Estudo transversal descritivo.

$\mathrm{N}=272$ crianças de 0-71 meses. triciptal. escolares chilenos)

Idade: 5-22 anos

Região: urbana e rural P.C.(Tanner)

Estudo transversal descritivo. de Newcastle Idade média - 5,5 anos.

Estudo transversal descritivo.
Amostra: $\mathrm{N}=17$ crianças desnutri-

Controle: $\mathrm{N}=14$ crianças nutridas.

Idade 6-24 meses hospitalizadas

Avaliação DNM* (Griffiths Mental

Antropometria: P.C., peso e es-

$\mathrm{N}=353$ crianças beduínas e 302

Antropometria: P.C., peso e altura.

Os 2 grupos vivem na mesma região, com hábitos culturais dife-

Judeus = classe média baixa da

Beduínos = semi-nômades, vivem em tendas, cabanas ou acampa-

As crianças apresentaram:

$41 \%$ - desnutrição crônica

Antropometria: peso, altura, P.C., 12\% - desnutrição aguda circunferência braquial e prega Pronunciado déficit no P.C.

$\mathrm{N}=4.500$ escolares $(38 \%$ dos

Avaliação do estado nutricional, $\mathrm{N}=219$ crianças de áreas pobres

$\mathrm{N}=1016$ crianças de Oxfordshire

Antropometria: peso, altura e P.C.

$\mathrm{N}=4.346$ escolares, de 5-18 anos.

Medida de P.C. (comparação com as curvas de Tanner, NeIhaus e Roche)

$\mathrm{NSE}^{\star *}$ - alto, médio e baixo (Escala de Graffar) ano de idade. ção urbana. maior na população rural. nores que as de Oxfordshire. ção às referências usadas.
Inicialmente, o grupo das crianças desnutridas estava muito abaixo dos controles. Após 36 meses de observação, os desnutridos mantinham padrões mais baixos, porém com diferenças menores em relação aos controles. Quanto ao peso/estatura, as crianças desnutridas apresentaram "catch-up" 1

As crianças beduínas nascem As práticas alimentares, como a peso levemente inferior ao amamentação exclusiva e prodas judias. Apresentam progressi- longada, o consumo escasso de vamente menor peso, estatura e carne e ovos, sugerem que, além P.C., sendo as diferenças estatis- do aporte calórico, outros fatores ticamente significativas com 1 contribuem para a desnutrição nas crianças beduínas. Os autores enfatizam que mesmo uma malnutrição leve pode levar a déficits importantes de crescimento, afetando até mesmo o P.C.

O déficit no P.C., nas crianças maiores reflete a restrição ao crescimento durante os 2 primeiros anos de vida.

Os autores sugerem a promoção de um melhor crescimento somático antes do $2^{\circ}$ ano, para um bom crescimento cerebral.

Em relação ao peso, a população rural apresentou $47,4 \%$ de desnutrição contra $26,2 \%$ da popula-

P.C. subótimo (valores abaixo da média para idade), foi 3 vezes

Os escolares da área rural apresentam níveis de desnutrição significantemente maiores que os da área urbana. Destacam-se as diferenças no P.C., pois têm-se apontado como o valor antropométrico mais sensível da desnutrição precoce e que está correlacionado com o peso do cérebro.

As crianças de Newcastle apre- Os autores comentam que dos sentaram todos os parâmetros an- valores antropométricos afetados tropométricos significantemente me- o que suscita mais preocupação

é o P.C., porque, a medida tem sido citada como preditor do futuro intelectual.

Os escolares de NSE** baixo Sendo o P.C. indicador da históapresentam P.C. menor em relaria nutricional, é possível afirmar que os escolares de NSE ${ }^{* *}$ baixo sofreram insultos nutricionais precoces.

* DNM = desenvolvimento neuromotor

${ }^{* *} \mathrm{NSE}=$ nível sócio-econômico 
Tabela IIA - Sumário de estudos que correlacionam perímetro cefálico e desenvolvimento neuromotor

\begin{tabular}{|c|c|c|c|}
\hline Autores & Métodos & Resultados & Conclusões \\
\hline $\begin{array}{l}\text { Gross et al. }^{(63)}(1978) \\
\text { New York }\end{array}$ & $\begin{array}{l}\text { Estudo longitudinal. } \\
\mathrm{N}=177 \text { crianças que nasceram } \\
\text { com peso = ou < } 2000 \mathrm{gr} \text {. } \\
\text { Medida de P.C. no R.N. (micro < } \\
\text { per } 10 \text {-Lubchenco). } \\
\text { "Status" neurológico no R.N. } \\
\text { (anormal-hipo ou hipertonia, letar- } \\
\text { gia, transiluminação, anormal). } \\
\text { Seguimento } 5 \text { anos e reavalia- } \\
\text { ção neurológica. }\end{array}$ & $\begin{array}{l}18 \% \text { com microcefalia ao nas- } \\
\text { cimento. } \\
45 \% \text { com P.C. entre percentil } 10-25 \text {. } \\
21 \% \text { com P.C. entre percentil } 26-10 \text {. } \\
11 \% \text { com P.C. > percentil } 50 \text {. } \\
53 \% \text { desnutridos intra-útero. } \\
\text { Anormalidade neurológica aos } 5 \\
\text { anos, correlacionada com mi- } \\
\text { crocefalia ao nascer. }\end{array}$ & $\begin{array}{l}\text { P.C. ao nascimento, correlacio- } \\
\text { nado com QI aos } 5 \text { anos. } \\
\text { A média de QI para as crianças } \\
\text { que eram PIG }{ }^{2} \text { e microcefálicas, } \\
\text { foi menor, quando comparadas } \\
\text { com o grupo PIG, com P.C. nor- } \\
\text { mal. } \\
\text { P.C. pequeno correlacionado } \\
\text { com desnutrição intra-útero, bai- } \\
\text { xo NSE } \text { N }^{5} \text {, Apgar baixo. } \\
\text { No estudo, não fica definida a } \\
\text { causa da microcefalia. }\end{array}$ \\
\hline $\begin{array}{l}\text { Ounsted et al. } \\
\text { (1984) Oxford }\end{array}$ & $\begin{array}{l}\text { Estudo longitudinal. } \\
\mathrm{N}=138 \mathrm{PIG}, 138 \mathrm{AIG}^{3} \text { e } 136 \mathrm{GIG}^{4} \text {. } \\
\text { Seguimento do nascimento aos } 7 \\
\text { anos. } \\
\text { Antropometria: peso, altura, P.C., } \\
\text { prega triciptal e prega subesca- } \\
\text { pular. } \\
\text { Avaliação neurológica: coorde- } \\
\text { nação motora, habilidade verbal e } \\
\text { raciocínio prático. }\end{array}$ & $\begin{array}{l}\text { P.C. diretamente relacionado: } \\
\text { PIG-raciocínio prático. } \\
\text { GIG-raciocínio prático e habili- } \\
\text { dade verbal. }\end{array}$ & $\begin{array}{l}\text { Ao analisar outras variáveis de } \\
\text { confundimento, a performance } \\
\text { intelectual está correlacionada } \\
\text { com NSE, principalmente entre } \\
\text { PIG. As meninas apresentam } \\
\text { melhor coordenação motora que } \\
\text { os meninos. Aos } 4 \text { anos, os fi- } \\
\text { Ihos de mães fumantes apre- } \\
\text { sentam pior performance (em } \\
\text { habilidade, coordenação motora } \\
\text { e fala). Aos } 7 \text { anos, os efeitos do } \\
\text { fumo diminuem, persistindo falha } \\
\text { em raciocínio prático. }\end{array}$ \\
\hline $\begin{array}{l}\text { Walther et al. }{ }^{(69)} \\
\text { (1988) Irlanda }\end{array}$ & $\begin{array}{l}\text { Estudo longitudinal. } \\
\mathrm{N}=25 \text { PIG, } 25 \text { AIG (grupo con- } \\
\text { trole). } \\
\text { Seguimento do nascimento aos } 7 \\
\text { anos. } \\
\text { Antropometria: peso, altura, P.C., } \\
\text { índice ponderal. } \\
\text { Avaliação comportamental: ati- } \\
\text { vidade, birra, timidez, irritabili- } \\
\text { dade, medo, concentração e } \\
\text { agressividade. } \\
\text { Avaliação acadêmica: desen- } \\
\text { volvimento cognitivo, sociabili- } \\
\text { dade e habilidade motoras. }\end{array}$ & $\begin{array}{l}\text { P.C. aos } 7 \text { anos significativa- } \\
\text { mente menor que o grupo con- } \\
\text { trole. } \\
\text { PIG- apresentam maior números } \\
\text { de problemas comportamentais e } \\
\text { acadêmicos, comparados com } \\
\text { AIG, aos } 7 \text { anos. }\end{array}$ & $\begin{array}{l}\text { As crianças PIG, aos } 7 \text { anos, } \\
\text { apresentam P.C. pequeno, baixo } \\
\text { índice ponderal e baixo pe- } \\
\text { so/altura. Problemas de com- } \\
\text { portamento, relacionados com } \\
\text { leves alteração neurológicas. } \\
\text { Estes achados, que haviam sido } \\
\text { detectados aos } 3 \text { anos, sugerem } \\
\text { que este padrão, pode persistir } \\
\text { em idades posteriores. }\end{array}$ \\
\hline $\begin{array}{l}\text { Simon et al. }{ }^{(70)}(1993) \\
\text { Alabama }\end{array}$ & $\begin{array}{l}\text { Estudo retrospectivo. } \\
\mathrm{N}=48<1500 \text { R.N.- peso (AIG). } \\
\text { Idade gestacional: média de } 28,5 \\
\text { semanas. } \\
\text { R.N. sem complicações. } \\
\text { A idade do "catch-up" para P.C. } \\
\text { foi determinada em cada criança. } \\
\text { As crianças sofreram avaliações } \\
\text { neurológica, periódicas, até } 12 \\
\text { meses. }\end{array}$ & $\begin{array}{l}\text { Houve uma notável correlação } \\
\text { entre o "catch-up" para P.C. e } \\
\text { resolução de problemas neu- } \\
\text { rológicos dos } 6 \text { ao } 12 \text { meses } \\
\text { (idade corrigida). }\end{array}$ & $\begin{array}{l}\text { As crianças prematuras que al- } \\
\text { cançaram o percentil } 5 \text { para P.C., } \\
\text { aproximadamente aos } 6 \text { meses, } \\
\text { mostram menos problemas neu- } \\
\text { romotores, que as do grupo de } \\
\text { prematuros que apresentam } \\
\text { "catch-up" mais tardio. O P.C. } \\
\text { pode ser a melhor medida para } \\
\text { monitorar a velocidade do cres- } \\
\text { cimento cerebral e para triagem } \\
\text { de crianças de risco. }\end{array}$ \\
\hline $\begin{array}{l}1 \text { - coeficiente de inte } \\
2 \text { - pequeno para a io } \\
3 \text { - adequado para a } \\
4 \text { - grande para a ida } \\
5 \text { - nível sócio-econô } \\
6 \text { - desenvolvimento }\end{array}$ & $\begin{array}{l}\text { gência } \\
\text { de gestacional } \\
\text { ade gestacional } \\
\text { gestacional } \\
\text { ico } \\
\text { uromotor }\end{array}$ & & \\
\hline
\end{tabular}


Tabela IIB - Sumário de estudos que correlacionam perímetro cefálico e desenvolvimento neuromotor

\begin{tabular}{|c|c|c|c|}
\hline Autores & Métodos & Resultados & Conclusões \\
\hline $\begin{array}{l}\text { Powell et al. }{ }^{(71)} \text { (1995) } \\
\text { Jamaica }\end{array}$ & $\begin{array}{l}\text { Estudo de intervenção. } \\
\mathrm{N}=127 \text { crianças } 9-24 \text { meses (com } \\
\text { desnutrição crônica). } \\
\text { Divididas em } 4 \text { grupos: controle, } \\
\text { com suplementação, com esti- } \\
\text { mulação e com ambos. } \\
\text { Antropometria: peso comprimento } \\
\text { ou estatura e P.C. } \\
\text { Avaliação do DNM }{ }^{6} \text { : locomotor, } \\
\text { coordenação manual-visual, audi- } \\
\text { ção, fala e performance. } \\
\text { Trabalho e Ql maternos, NSE, } \\
\text { ordem de nascimento da criança. }\end{array}$ & $\begin{array}{l}\text { P.C. estava altamente correla- } \\
\text { cionado com a idade mental so- } \\
\text { mente na época da inscrição. } \\
\text { Após a intervenção, o melhor } \\
\text { DNM estava associado com a } \\
\text { estatura. }\end{array}$ & $\begin{array}{l}\text { Provavelmente existem muitos } \\
\text { fatores que interferem na relação } \\
\text { crescimento - DNM, um resulta- } \\
\text { do importante e inesperado foi } \\
\text { que aproximadamente } 2 / 3 \text { apre- } \\
\text { sentaram melhora do DNM inde- } \\
\text { pendente das variações antropo- } \\
\text { métricas. Ao menos em parte, os } \\
\text { benefícios da suplementação in- } \\
\text { dependem de incrementos no cres- } \\
\text { cimento físico. O esclarecimento } \\
\text { desse mecanismo requer mais in- } \\
\text { vestigações. }\end{array}$ \\
\hline $\begin{array}{l}\text { Ivanovic et al. }{ }^{(66)} \\
\text { (1996) Chile }\end{array}$ & $\begin{array}{l}\text { Estudo transversal descritivo. } \\
\mathrm{N}=4.509 \text { escolares ( } 1^{\circ} \text { e } 2^{\circ} \text { grau) } \\
\text { idade 6-17 anos. } \\
\text { Estado nutricional: peso, altura, } \\
\text { P.C., altura sentada, circunfe- } \\
\text { rência braquial e pregas cutâ- } \\
\text { neas. } \\
\text { NSE (Escala de Graffar), avaliação } \\
\text { de aprendizado escolar. }\end{array}$ & $\begin{array}{l}\text { O aprendizado foi correlacionado } \\
\text { com o P.C.. Este resultado foi } \\
\text { encontrado em todos os níveis } \\
\text { sócio-econômicos e aumentou a } \\
\text { correlação com a progressão da } \\
\text { criança no sistema escolar. En- } \\
\text { tretanto, crianças com P.C. subó- } \\
\text { timo (qualquer valor abaixo da } \\
\text { média), de NSE mais elevado } \\
\text { apresentaram melhor aprendiza- } \\
\text { do, quando comparadas com as } \\
\text { de níveis mais baixos. }\end{array}$ & $\begin{array}{l}\text { Sendo o P.C., um indicador de } \\
\text { desnutrição pregressa, e do de- } \\
\text { senvolvimento cerebral, o estudo } \\
\text { sugere que crianças com P.C. } \\
\text { abaixo da média para a idade, } \\
\text { sofreram desnutrição no } 1^{\circ} \text { ano } \\
\text { de vida, provocando um cresci- } \\
\text { mento cerebral lento e baixos } \\
\text { coeficientes de inteligência. }\end{array}$ \\
\hline $\begin{array}{l}2 \text { - pequeno para a id } \\
3 \text { - adequado para a } \\
4 \text { - grande para a ida } \\
5 \text { - nível sócio econôr } \\
6 \text { - desenvolvimento }\end{array}$ & $\begin{array}{l}\text { ência } \\
\text { de gestacional } \\
\text { ade gestacional } \\
\text { gestacional } \\
\text { co } \\
\text { uromotor }\end{array}$ & & \\
\hline
\end{tabular}

pois as condições sócio-econômicas desfavoráveis potencializam seus efeitos deletérios. Torna-se evidente que a criança desnutrida e sem escola (falta de estímulo) é a que se encontra em pior situação( ${ }^{(75)}$.

As crianças com desnutrição grave apresentam a cabeça pequena ${ }^{(76)}$ e EEG alterado durante, pelo menos, um ano após o episódio(77).

MORLEY \& LUCAS, $1997^{(78)}$, através de revisão de estudos em que procuram esclarecer a influência da desnutrição precoce e a função cognitiva, sugerem que, além dos déficits protéicos e energéticos e de oligoelementos (ferro e zinco principalmente) os ácidos graxos, polissaturados, de cadeia longa, mais notadamente o ácido docohexanóico, presente no leite humano, são nutrientes fundamentais para o desenvolvimento do sistema nervoso.
A importância da desnutrição precoce no desenvolvimento cognitivo posterior, continua em debate e necessita de maiores investigações, para um total esclarecimento dos mecanismos fisiopatológicos.

\section{PERÍMETRO CEFÁlICO NA INFÂNCIA E RE- FLEXOS NA VIDA ADULTA}

BARKER et al., $1993^{(79)}$, apresentaram um estudo de seguimento, de uma amostra de mil, quinhentos e oitenta e seis (1586) homens, nascidos entre 1907 e 1924, em Sheffield, Inglaterra, onde rotineiramente eram obtidos o perímetro cefálico, comprimento e idade gestacional. Os autores demonstraram a queda das taxas por doenças cardiovasculares com o aumento do peso ao nascer. O estudo mostra que homens com 
menor perímetro cefálico ou menor peso ao nascer, ou ambos, têm maior risco de morrer em decorrência de doenças cardiovasculares, antes dos sessenta e cinco (65) anos, quando comparados com os de perímetro cefálico maior. Os autores sugerem que um perímetro cefálico pequeno e baixo peso refletem o padrão de crescimento fetal associado com mudanças em certos tecidos, incluindo vasos sangüíneos, e pâncreas. Essas transformações "programariam" a pressão sangüínea, e o metabolismo da glicose e insulina, e as doenças cardiovasculares na idade adulta. $\mathrm{O}$ estudo mostra, pela primeira vez, que a falha no crescimento fetal é seguida por um aumento nas taxas de morte por causas cardiovasculares, na idade adulta. BARKER et al., $1989^{(80)}$, já haviam relatado que a taxa de mortalidade por isquemia cardíaca, entre os indivíduos que apresentavam, com um (1) ano de vida, peso de 8.165 $\mathrm{g}$ ou menos, era três (3) vezes maior daqueles que apresentavam $12.247 \mathrm{~g}$ ou mais.

THAME et al., $1997^{(81)}$, apresentam estudo retrospectivo, realizado em 1990, na Jamaica. Da amostra composta de dois mil, trezentos e noventa e quatro (2.394) nascimentos, com duzentos (200) a trezentos e cinco (305) dias de gestação, foram obtidos dados referentes ao peso ao nascer, perímetro cefálico, comprimento, índice ponderal, peso da placenta, além de cálculo dos índices: perímetro cefálico/comprimento e peso da placenta/peso ao nascer. Para caracterizar o estado nutricional materno, foram obtidos: peso, altura e concentração de hemoglobina. Os autores demonstraram que mulheres com baixo índice de massa corporal, têm filhos menores, e com perímetro cefálico menor. A concentração de hemoglobina parece apresentar dois efeitos sobre o crescimento fetal: no início da gestação, reflete o estado nutricional materno, porém, a queda no índice ponderal e uma hemoglobina que excede $12,5 \mathrm{~g} / \mathrm{dl}$ apontam para uma disfunção circulatória materna. A hemodiluição é normal durante a gravidez. Mulheres desnutridas são incapazes de apresentar um hemodiluição adequada, e o volume de plasma reduzido se associa a nascimentos de crianças pequenas. A anemia $(\mathrm{Hb}<10,5 \mathrm{~g} / \mathrm{dl})$, no terceiro trimestre da gravidez, apresenta associação com baixo peso ao nascer, baixo índice ponderal e alto índice peso da placenta/peso ao nascer. Mulheres mal nutridas não conseguem ajustar as demandas metabólicas para um adequado desenvolvimento fetal, gerando filhos menores, com perímetro cefálico pequeno. Os autores ressaltam que o baixo peso ao nascer reflete a nutrição fetal e parece ser o primeiro marco para do- enças cardiovasculares crônicas, na idade adulta. Afirmam que é necessário investigar os mecanismos dos efeitos do estado nutricional materno no crescimento e no desenvolvimento fetal.

GRAVES et al., $1996^{(82)}$, apresentam um estudo transversal em uma amostra de mil, novecentos e oitenta e cinco (1985) indivíduos, no qual encontraram correlação estatisticamente positiva entre perímetro cefálico menor e gravidade da doença de Alzheimer. Desde 1968, através dos estudos feitos por TOMLINSON; BLESSED \& ROTH, $1968^{(83)}$, foi demonstrado que a diferença entre um cérebro sadio e um com a doença de Alzheimer é quantitativa, e não qualitativa. As pessoas que possuem cérebros maiores têm maior reserva cerebral, podendo sofrer maior número de insultos antes de manifestarem sinais clínicos da doença. Sabendo-se que o cérebro tem seu maior desenvolvimento da vida intra-uterina até os três (3) primeiros anos de vida, os autores sugerem que cuidados pré-natais e o controle da nutrição e condições de vida, nessa faixa etária, podem aumentar a reserva cerebral e, com isso, atrasar o início e diminuir a gravidade da doença de Alzheimer.

SCHOFIELD et al., $1997^{(84)}$, investigando a associação entre perímetro cefálico e Doença de Alzheimer, realizaram estudo transversal em uma amostra de seiscentos e quarenta e nove (649) sujeitos, submetidos a avaliações neurológicas, psicológicas e antropométricas. As análises estatísticas demonstraram que mulheres com perímetro cefálico abaixo do percentil 5 estavam 2,9 vezes mais predispostas a desenvolver a Doença de Alzheimer. Para os homens que apresentam os mesmos níveis de perímetro cefálico, o risco é 2,3 vezes maior em comparação com os que apresentam perímetro cefálico acima do percentil 5.

\section{CONCLUSÕES}

O perímetro cefálico é um parâmetro antropométrico altamente correlacionado com o tamanho cerebral. A desnutrição, nos primeiros anos de vida, leva a uma diminuição do tamanho corporal; entretanto, considerando que o crescimento continua até aproximadamente os dezoito (18) anos, se a nutrição for adequada mais tardiamente, o impacto negativo, inicial, poderá ser minimizado. Porém, este fato não ocorre com o cérebro, porque os dois (2) primeiros anos de vida não são somente o período de maior crescimento, mas constituem o período em que o crescimento cerebral se completa quase que totalmente $(83,6 \%$ no $1^{\circ}$ ano de vida). 
A antropometria do perímetro cefálico, após os dois (2) primeiros anos de vida, no pré-escolar, escolar e na idade adulta, reflete o estado nutricional do início da vida, e pode ser um preditor de saúde e qualidade de vida, apontando as populações de risco para um desenvolvimento neuromotor inadequado (caso não recebam estímulos compensatórios), para doenças cardiovasculares, diabetes e para o desenvolvimento mais precoce e grave da Doença de Alzheimer, em indivíduos predispostos. Tais fatos são de extrema importância para o desenvolvimento de políticas de saúde que visem prevenir a desnutrição precoce e suas consequiências.
Finalmente, pelas implicações acima, recomenda-se que a medida do perímetro cefálico, ao lado de outras medidas rotineiramente usadas, seja empregada na avaliação do estado nutricional de populações.

\section{AGRADECIMENTOS}

Os autores agradecem aos professores, Dra. Angélica Maria Bicudo Zeferino, Dr. Roberto Teixeira Mendes e Dr. Carlos Roberto Freire de Rivorêdo, as sugestões ao estudo.

MACCHIAVERNI LML \& BARROS FILHO AA. Head circumference: why always measure it. Medicina, Ribeirão Preto, 31: 595-609, oct./dec. 1998.

ABSTRACT: A review on the importance of head circumference anthropometry is present by authors in this study. It includes a description of the techniques and curves frequently used, the secular trends of the head circumference in different populations, its correlation to the size of the brain and the characteristics of cerebral growth.

Cerebral growth differs from that of the other parts of the body $-83,6 \%$ of its growth takes place within the first year of life and therefore the head circumference $(\mathrm{HC})$ is an anthropometric measure that could provide information regarding the first two years of an individual's life. The vulnerable periods of the human brain with regard to nutrition can be seen by the sequels left due to early malnutrition - the reduction in the cerebral mass, a reduced number of glia cells and Purkinje's cerebellar granular cells and a reduction in the size of network, ramifications and the number of synapses. The authors used these evidences to study populations that suffered malnutrition during the first two years of life and show that the development of the neuro-motor potential was hampered and that it worsens or improves according to the environment.

Recent studies suggest that malnutrition during the early years of life is correlated to an increase in cardiovascular diseases at adult stage as a result of adaptation mechanisms used by the mother and infant to face the nutritional situation. A head circumference below normal standards is a risk factor for the onset of Alzheimer's disease in adults who have a predisposition to this disease.

The authors recommend that the measure of the head circumference, should be included together with other anthropometric measures in studies that evaluate the health and nutrition of population.

UNITERMS: Cephalometry. Child Nutrition Disorders. Child Development. Public Health. Anthropometry.

\section{REFERÊNCIAS BIBLIOGRÁFICAS}

1 - WORLD HEALTH ORGANIZATION. An evaluation of infant growth: the use and interpretation of anthropometry in infants. Bull World Heath Organ 73: 165-174,1995.

2 - YARBROUGH $C$ et al. Anthropometry as an index of nutritional status. In: ROCHE AF \& FALKNER F, eds. Nutrition and malnutrition: Identification and measurement, Plenum Press, New York, p. 15-16, 1974

3 - STOCH MB \& SMYTHE PM. 15-year development study on effects of severe undernutrition during infancy on subsequent physical growth an intellectual functioning. Arch Dis Child 51: 327-335, 1976.
4 - WEAVER DD \& CHRISTIAN JC. Familial variation of head size and adjustment for parental head circumference. J Pediatr 96: 990-994, 1980.

5 - ROCHE AF et al. Head circumference data: birth to 18 years. Pediatrics 79: 706-712, 1987.

6 - JELLIFFE DB \& PATRICE JELLIFFE EF. Anthropometry: major measurements. In: JELLIFFE DB \& PATRICE JELLIFFE EF. Community nutritional assessment, Oxford University Press, Oxford, p. 68-105, 1989.

7 - IVANOVIC DM et al. Head circumference of Chilean school children aged 5 to 18 years and socioeconomic status. Rev Med Chil 123: 587-599, 1995. 
8 - SMITH DW. Measurements, methods, and standars. In: SMITH DW. Growth and its disorders, W.B. Saunders, Philadelphia, p.18-61, 1977.

9 - CAMERON N. Anthropometric Measurements. In: CAMERON N. The measurement of human growth, Coom Helm, London: p.56-99, 1984.

10 - WORLD HEALTH ORGANIZATION. Measuring nutritional status, Geneva, WHO, 1983.

11 - ORGANIZACIÓN PANAMERICANA DE LA SALUD. Salud maternoinfantil y atención primária en las Américas: hechos y tendencias. Publ. Cient. 461, 1984.

12 - KANAWATI AA \& MACLAREN DS. Assessment of marginal nutrition. Nature 228: 573-575, 1970.

13 - ZERFAS AJ. Anthropometric field methods: general. In: JELLIFFE DB \& JELLIFFE EF, eds. Human nutrition- A comprehensive treatise. Plenum Press, NewYork, v. 2, p.339-364, 1979.

14 - MALINA RM et al. Head and chest circumference in rural Guatemala Ladino children, birth to seven years of age. Am J Clin Nutr 28: 1061-1070, 1975.

15 - SPURR GB; REINA JC \& BARAC-NIETO M. Marginal malnutrition in school aged Colombian boys: anthropometry and maturation. Am J Clin Nutr 37: 119-132, 1983.

16 - WORLD HEALTH ORGANIZATION. PHYSICAL STATUS: The use and interpretation of anthropometry. World Health Organ Tech Rep Ser 854, Geneva, 1995.

17 - NELHAUS G. Head circumference from birth to eigteen years. Practical international and interracial graphs. Pediatrics 41: 106-114, 1968.

18 - MEREDITH HV. Human head circumference from birth to early adulthood: racial, regional and sex comparions. Growth 35: 233-251, 1971.

19 - BABSON SG. Growth of low-birth-weight infants. J Pediatr 77: 11-18, 1970.

20 - BHARGAVA SK et al. Growth and sexual maturation of low birth weight children: a 14 years follow up. Indian Pediatr 32: 963-970, 1995.

21 - ROCHE AF; MUKHERJEE D \& GUO S. Head circumference growth patterns: Birth to 18 years. Hum Biol 58: 893906, 1986.

22 - ISHIKAWA T et al. Growth in head circumference from birth to fifteen years of age in Japan. Acta Paediatr Scand 76: 824-828, 1987.

23 - OUNSTED M; MOAR VA \& SCOTT A. Head circumference charts updated. Arch Dis Child 60: 936-939, 1985.

24 - JAFFE M et al. Variability in head circumference growth rate during the first 2 years of life. Pediatrics 90: 190-192, 1992.

25 - MARCONDES E \& MARQUES RM. Crescimento e desenvolvimento pubertário em crianças e adolescentes brasileiros. III- Perímetro cefálico e torácico, Ed. Brasileira de Ciências, São Paulo,1983, 24 p.

26 - PUCCIARELLI HM, CARNESE FR \& GUIMAREY LM. Desnutrición y dimorfismo sexual. Cienc Hoy 6: 53-59, 1996.

27 - WINGER JMA; SHEN EJ \& SOLOMON IL. Growth standars in the first two years of life based on measurements of white and black children in a prepaid health care program. Pediatrics 47: 818-825, 1971.
28 - DUNCAN B; SMITH NA \& BRIESE FW. A comparison of growth: spanish-surnamed with non-spanish-surnamed children. Am J Public Health 69: 903-907, 1979.

29 - MONTEIRO CA. Velhos e novos males da saúde no Brasil: A evolução do país e suas doenças, Editora Hucitec/ NUPENS/ USP, São Paulo 1995. 359p

30 - HAUSPIE RC; VERCAUTEREN M \& SUSANNE C. Secular changes in growth and maturation: an update. Acta Paediatr 86: 20-27, 1997. Suppl. 423

31 - TANNER JM. Growth as mirror of condition of society: secular trends and class distictions. Acta Paediatr Jpn 29: 96-103, 1987.

32 - DAVIES DP; LEUNG SF \& LAU SP. Secular trends in head growth (letter). Arch Dis Child 61: 632-624, 1986.

33 - GRAHAM CG. Effect of infantile malnutrition in growth. Fed Proc 26: 139-143, 1967.

34 - EICHORN DH \& BAYLEY N. Growth in head circumference from birth through young adulthood. Child Dev 33: 257-271, 1962.

35 - JELLIFFE DB. The assessment of nutritional status of community. World Health Organ Monogr Geneve 53: 69-70, 1966.

36 - WINICK M \& ROSSO P. Head circumference and intellectual growth of the brain in normal and marasmic children. $\mathbf{J}$ Pediatr 74: 774-778, 1969.

37 - GUIMAREY LM. Crescimento e estado nutricional em escolares de Paulínia-São Paulo- Brasil. Tese de Doutorado, Faculdade de Ciências Médicas da UNICAMP, Campinas. p. 1-100, 1983.

38 - GONZÁLES-RICHMOND JA. La antropometria en la avaluación del estado nutricional. Bol Méd Hosp Infant México 42: 207-212, 1985.

39 - MORCILLO AM. Estudo comparativo de sete parâmetros antropométricos em escolares da cidade de Paulínia- São Paulo, referente aos períodos de 1979/1980 e 1984/1985. Tese de Doutorado, Faculdade de Ciências Médicas da UNICAMP, Campinas. p.1-124, 1987.

40 - DOBBING J. The later development of the brain and its vulnerability. In: DAVIS J \& DOBBING J, eds. Scientific foundations of paediatrics, Heineman, London, p.744-759, 1981.

41 - BEDI KS. Lasting neuro-anatomical changes following undernutrition. In: DOBBING J, ed. Early nutrition and later achievement, Academic Press, London, p.1-49, 1987.

42 - GRANTHAM-MCGREGOR SM. Efecto de la malnutrición en el desarrolo mental. In: WATERLOW JC, ed. Malnutrición Proteico-Energética, Publ Cient 555: p. 423-443, 1996.

43 - BJERRE I. Physical growth of 5-year-old children with a low birth weight. Acta Paediatr Scand 64: 34-43, 1975.

44 - OUNSTED M; MOAR V \& SCOTT A. Growth in the first four years: II. Diversity within of small-for-dates and largefor-dates babies. Early Hum Dev 7: 29-39, 1982.

45 - SANN L et al. Effects of prematurity and dysmaturity on growth at age 5 years. J Pediatr 109: 681-686, 1986.

46 - RIZZARDINI MP et al. Crecimiento postnatal del recién nacido de muy bajo peso (RNMBP) Antropometría a tres años plazo, estudio longitudinal. Rev Chil Pediatr 62: 285-289, 1991.

47 - HACK M et al. Health of very low birth weight children during their first eight years. J Pediatr 122: 887-892, 1993. 
48 - XAVIER CC et al. Crescimento de recém-nascidos pré-termo. J Pediatr 71: 22-27, 1995.

49 - LUBCHENCO LO et al. Intrauterine growth as estimated from liveborn birth-weight data at 24 to 42 weeks of gestation. Pediatrics 32: 793-800, 1963.

50 - FUJIMURA M. Factors which influence the timing of maximum growth rate of head in low birthweight infants. Arch Dis Child 52: 113-117, 1977.

51 - LECHTIG A \& KLEIN RE. Effect of food supplementation during pregnancy and lactation on infant mortality, morbidity, and physical growth. Arch Latinoam Nutr 24: 99-142, 1979. Suppl 1.

52 - DESAI ID et al. Anthropometric and cycloergometric assessment of nutritional status of children of agricultural migrant workers in Southern Brazil. Am J Clin Nutr 34: 1925-1934, 1981.

53 - GRANTHAM-MCGREGOR SM et al. Longitudinal study of growth and development of young Jamaican children recovering from severe protein-energy malnutrition. Dev Med Child Neurol 24: 321-331, 1982.

54 - DAGAN R et al. Growth and nutritional status of Beduin infants in the Negev Desert, Israel: evidence for marked stunting in the presence of only mild malnutrition. Am J Clin Nutr 38: 747-756, 1983

55 - SERENIUS F; FOUGEROUSE D \& SEBAI Z. Growth and nutritional status of rural preschool children in Saudi Arabia. Acta Paediatr Scand p.104-120, 1988. Suppl. 346

56 - IVANOVIC RM; OLIVARES MG \& IVANOVIC DM. Estado nutricional en escolares chilenos urbanos y rurales de la Región Metropolitana, 1986-1987. Rev Chil Pediatr 61: 210-217, 1990.

57 - WRIGHT CM et al. A comparison of height, weight and head circumference of primary school children living in deprived and non-deprived circumstances. Early Hum Dev 31: 157162, 1992.

58 - BOHLER E \& BERGSTRÖM S. Frequent diarrhoeas in early childhood have sustained effects on height, weight and head circumference of children in East Bhutan. Acta Paediatr 85: 26-30, 1996.

59 - GHOSAL S et al. Disproportionate head growth retardation in cystic fibrosis. Arch Dis Child 72: 150-152, 1995.

60 - VRLENICK LA et al. The effect bronchopulmonary dysplasia on growth at school age. Pediatrics 95: 855-859, 1995.

61 - FINE RN; SALUSKY IB \& ETTINGER RB. The therapeutic approach to the infant, child, and adolescent with end-stage renal disease. Pediatr Clin North Am 34: 789-801, 1987.

62 - APPLETON RE et al. Decline in head growth and cognitive impairment in survivors of acute lymphoblastic leukaemia. Arch Dis Child 65: 530-534, 1990.

63 - GROSS SJ; KOSMETATOS N \& GRIMES CT. Newborn head size and neurological status. Am J Dis Child 132: 753756, 1978.

64 - CRAVIOTO J. Desnutrição e desenvolvimento mental. In: Resumos, XXXVI Seminário Nestlé de Nutrição "A alimentação na infância e suas conseqüências a longo prazo", Filipinas, p. 4-7, 1994.

65 - NELSON KB \& DEUTSHBERGER J. Head size as a predictor of four year IQ. Dev Med Child Neurol 12: 487-495, 1970.
66 - IVANOVIC DM. Nutrition and learning in Chilean school age children: Chile's Metropolitan Region. Survey 1986-1987. Nutrition 12: 321-328, 1996.

67 - ROSADO MLC et al. Crescimento craniano na criança. Arq Neuropsiquiatr 47: 397-402, 1989.

68 - OUNSTED MK; MOAR VA \& SCOTT A. Children of deviant birthweight at the age of seven years: health, handcap, size and developmental status. Early Hum Dev 9: 323-340, 1984.

69 - WALTHER FJ. Growth and development of term disproportinate small-for-gestational age infants at the age of 7 years. Early Hum Dev 18: 1-11, 1988.

70 - SIMON PN; BRADY RN \& STAFFORD RL. Catch-up head growth and motor performance in very-low-birthweight infants. Clin Pediatr (Phila) 32: 405-411, 1993.

71 - POWELL CA et al. Relatioships between physical growth, mental development and nutritional supplementation in stunted children: the Jamaican study. Acta Paediatr 84: 22 29, 1995.

72 - POLLIT E; HAAS J \& LEVITSKY D. International conference on iron deficiency and behavioral development. Am J Clin Nutr 50: 566-705, 1989. Suppl.

73 - ENGSNER G et al. Brain growth in children with kwashiorkor. Acta Paediatr Scand 63: 687-694, 1974.

74 - WALKER SP et al. Nutritional supplementation, psychosocial stimulation, and growth of stunted children: the Jamaica study. Am J Clin Nutr 54: 642-648, 1991.

75 - POLLIT E. Desnutricion y rendimiento escolar. Crecimiento y desarrollo - Hechos y tendencias, Publ Cient 510: 324348, 1988.

76 - BROWN RE. Decreased brain weight in malnutrition and its implications. East Afr Med J 42: 584-595, 1965.

77 - BARNET AB \& WEISS AP. Abnormal auditory evoked potentials in early infancy malnutrition. Science 201: 450-452, 1978.

78 - MORLEY R \& LUCAS A. Nutrition and cognitive development. Br Med Bull 53: 123-134, 1997.

79 - BARKER DJP et al. The relation of small head circumference and thinness at birth to death from cardiovascular disease in adult life. BMJ 306: 422-426, 1993.

80 - BARKER DJP et al. Weight in infancy and death from ischaemic heart disease. Lancet II: 577-580, 1989.

81 - THAME M et al. Relationship between maternal nutritional status and infant's weight and body proportions at birth. Eur J Clin Nutr 51: 134-138, 1997.

82 - GRAVES AB et al. Head circumference as a measure of cognitive reserve - Association with severity of impairment in Alzheimer's Disease. Br J Psychiatry 169: 86-92, 1996.

83 - TOMLINSON BE; BLESSED G \& ROTH M. Observations on brains of nondemented old people. J Neurol Sci 7: 331-356, 1968.

84 - SCHOFIELD PW et al. An association between head circumference and Alzheimer's disease in a population-based study of aging and dementia. Neurology 49: 30-37, 1997.

Recebido para publicação em 30/07/98

Aprovado para publicação em 10/11/98 